\title{
Le dualisme linguistique de Dionysios Solomos
}

L'italien et le grec dans la genèse des Libres Assiégés

\section{Kostis Pavlou}

\section{(2) OpenEdition}

1 Journals

\section{Édition électronique}

URL : http://journals.openedition.org/genesis/2406

DOI : $10.4000 /$ genesis. 2406

ISSN : 2268-1590

Éditeur :

Presses universitaires de Paris Sorbonne (PUPS), Société internationale de génétique artistique littéraire et scientifique (SIGALES)

\section{Édition imprimée}

Date de publication : 4 juin 2018

Pagination : 131-144

ISBN : 979-10-231-0604-6

ISSN : 1167-5101

\section{Référence électronique}

Kostis Pavlou, "Le dualisme linguistique de Dionysios Solomos », Genesis [En ligne], 46 | 2018, mis en ligne le 30 octobre 2018, consulté le 07 septembre 2019. URL : http://journals.openedition.org/ genesis/2406; DOI : 10.4000/genesis.2406 


\title{
Le dualisme linguistique de Dionysios Solomos L'italien et le grec dans la genèse des Libres Assiégés
}

\author{
Kostis Pavlou
}

In memoriam M. П.

$\mathrm{D}$ ans l'introduction à sa traduction en français de la poésie grecque de Dionysios Solomos publiée en 1945, Robert Levesque regrette le fait que le public en France - et en Europe en général - ignore non seulement l'œuvre de ce poète grec de premier plan, mais même son nom ${ }^{1}$. Malheureusement, cette situation n'a pas évolué depuis : à l'exception du public hellénophone, Solomos demeure toujours presque inconnu pour les lecteurs européens. Nous espérons le faire mieux connaître en publiant ici sous une forme inédite trois feuillets empruntés au dossier de genèse bilingue de son poème Les Libres

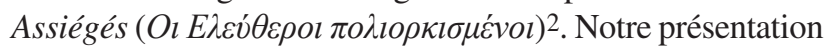
insistera sur les stratégies scripturaires qui révèlent une relation très complexe entre l'italien et le grec. Quelques précisions concernant le bilinguisme de Solomos et sa production littéraire seront nécessaires avant d'entrer dans le vif du sujet.

\section{Les langues de Solomos et sa création littéraire plurilingue : quelques repères fondamentaux}

Il faut rappeler, tout d'abord, que l'écriture de Solomos (Zante, 1798 - Corfou, 1857) est presque entièrement bilingue. Ce bilinguisme peut être attribué, d'une part, à la situation historique et sociale des îles Ioniennes et, d'autre part, à la biographie du poète, qui s'est formé en Italie où il a fait de longues études entre 1808 et 1818.

Les îles Ioniennes, ou l'Heptanèse (c'est-à-dire les SeptÎles), ont été progressivement conquises par la République de Venise à partir de la fin du XIVe siècle, et elles sont restées sous la domination vénitienne jusqu'en 1797, à savoir jusqu'à la dissolution de la Sérénissime par le traité de Campoformio. Après ce traité, le grec gagne progressivement du terrain au détriment de l'italien, qui avait pris beaucoup d'importance sur les îles. Cependant, bien que l'italien ait perdu son statut de langue officielle en 1852, les savants et les aristocrates des Sept-Îles ont continué à l'utiliser dans la plupart des situations de communication, du moins jusqu'à l'unification des îles avec la Grèce (1864) et, dans une certaine mesure, tout au long du XIXe siècle.

Par conséquent, le bilinguisme de Solomos au niveau individuel est un résultat du bilinguisme et du biculturalisme dominants dans l'Heptanèse. Étant le fils du comte Nikolaos Solomos, le poète a eu l'occasion d'approfondir la connaissance de la langue italienne dès son enfance. Suivant l'habitude du milieu aristocratique de l'Heptanèse, Solomos quitte son île natale en 1808 et part pour l'Italie, où il fait d'abord ses études secondaires au lycée de Crémone (1808-1815) et ensuite des études de droit à l'université de Pavie (1815-1818). Un si long séjour au pays de Dante et une formation reçue exclusivement en italien ont mis la langue grecque du poète «en sommeil» de sorte que, dès son retour à Zante, il devra la réapprendre. De plus, le grec de Solomos est en fait un idiolecte fondé essentiellement sur l'expression orale qui évolue en contact permanent avec l'italien et les autres langues (grec ancien, latin, français), dont on trouve les traces dans ses manuscrits.

Pour s'en tenir aux grandes lignes de la création littéraire plurilingue de Solomos, on peut rappeler que ses

1. Solomos : introduction, prose et poèmes, trad. du grec et éd. par Robert Levesque, Athènes, Icaros, «Collection de l'Institut français d'Athènes ", 1945, p. 9.

2. La totalité des autographes de Solomos parvenus jusqu'à nous ont été

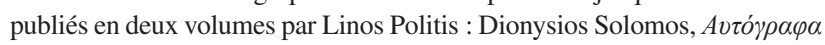
E $\rho \gamma \alpha$ (Euvres autographes), vol. I : Fac-similés; vol. II : Transcription typographique (introductions et notes en grec), Thessalonique, 1964. Dorénavant, l'abréviation $A E$ renvoie à cette édition; comme c'est l'habitude dans les études solomiennes, cette abréviation est suivie par l'indication de la page et de la ligne (l'astérisque [*] désigne l'interligne). 
premières et ses dernières tentatives poétiques (environ 1818-1822 et 1849-1855) ont été effectuées en italien. Pourtant, la quintessence de son écriture correspond à la longue phase intermédiaire de sa carrière poétique (environ 1824-1851). Dans cette période de maturité, caractérisée par une production en langue grecque, Solomos compose ses œuvres romantiques majeures qui restent en grande partie

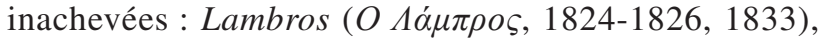

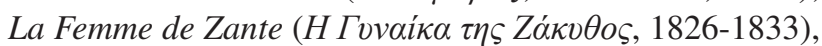

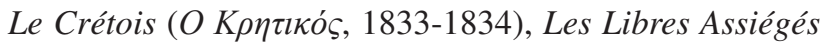

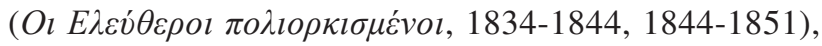

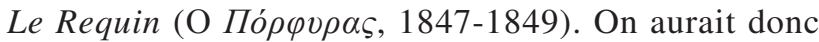
pu s'attendre à ce que l'usage du grec prédomine dans cette période ${ }^{3}$. Mais en réalité, Solomos met en œuvre une méthode de composition originale, qui est constituée par le va-et-vient incessant entre l'italien et le grec, comme en témoignent ses documents de travail bien conservés 4 .

Nous présentons ici un exemple concret de cette interaction des langues dans la genèse poétique chez Solomos à partir de trois pages manuscrites de son œuvre Les Libres Assiégés, caractéristique de sa période de maturité. Au risque de simplifier, on peut affirmer que l'italien est la langue des plans, des scénarios et des esquisses, alors que la composition poétique se fait en langue grecque; mais, comme nous le verrons, des interférences plus profondes et plus complexes ont lieu entre les deux langues.

\section{Le premier projet d'écriture des Libres Assiégés}

Le premier projet des Libres Assiégés a été rédigé approximativement entre 1834 et 1844 , en distiques décapentasyllabiques ${ }^{5}$, et le deuxième à peu près entre 1844 et 1851 , en décapentasyllabes non rimés. Les trois pages manuscrites que nous étudierons proviennent précisément du premier projet d'écriture de l'œuvre dont le sujet est le siège et la chute de Missolonghi (15 mars 1825-10 avril 1826). Cet événement, qui compte parmi les plus héroïques de la guerre d'indépendance grecque (1821-1829), ne constitue pour Solomos que le cadre général pour construire une œuvre au caractère philosophique et transhistorique : d'une part, les «libres assiégés » sont bien entendu les combattants de Missolonghi, mais cet oxymore est avant tout une métaphore de la condition humaine; d'autre part, les événements historiques deviennent porteurs de significations symboliques universelles.

\section{L'examen des trois pages manuscrites}

\section{Une brève note sur les illustrations}

Chacune des trois pages manuscrites ${ }^{6}$ est présentée par un fac-similé, une transcription diplomatique portant un repérage des campagnes d'écriture (désormais c.e.), une transcription quasi diplomatique dans laquelle les blocs d'écriture en italien et en grec sont traduits en français. Les fac-similés (voir fig. 1, 4, 7) correspondent à ceux de l'édition des autographes par Linos Politis 7 . Les transcriptions diplomatiques (voir fig. 3, 6, 9) sont inédites, et ont pour objectif le déchiffrement du manuscrit en même temps qu'une représentation aussi exacte que possible de sa topographie ${ }^{8}$. Le repérage des c.e. a comme objet principal

3. Cependant, il serait erroné d'affirmer que la création littéraire grecque de Solomos ne commence qu'en 1824, étant donné qu'il existe également des compositions du poète en langue grecque de la période allant de 1818 à 1823. Il s'agit soit de petits poèmes [par exemple, Ode à la Lune ( $\Omega \delta \dot{\eta} \varepsilon l \varsigma \tau \eta$

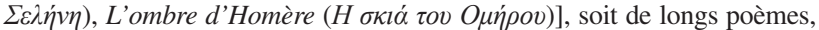

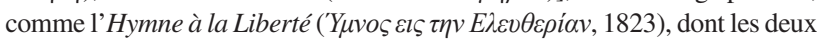
premières strophes constituent l'hymne national de la Grèce depuis 1865 . Notons que, contrairement aux œuvres romantiques de la période de maturité, les poèmes de cette première production grecque de Solomos s'inscrivent largement dans une esthétique néo-classique et préromantique.

4. Les manuscrits de Solomos sont conservés dans trois institutions grecques : 1'Académie d'Athènes, la Bibliothèque nationale de Grèce (Athènes) et le Musée de Solomos et d'éminentes personnalités de Zante (Zante).

5. Le décapentasyllabe est le vers de quinze syllabes typique de la production populaire byzantine et néo-hellénique, apte à servir un poème possédant une puissance narrative comme Les Libres Assiégés.

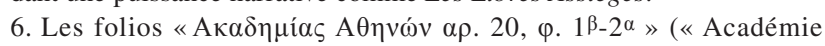
$\mathrm{d}^{\prime}$ 'Athènes $\mathrm{n}^{\mathrm{o}} 20,1 \mathrm{v}^{\mathrm{o}}-2 \mathrm{r}^{\mathrm{o}}$ », dorénavant fos $\mathrm{AA} 20,1 \mathrm{v}^{\mathrm{o}}-2 \mathrm{r}^{\mathrm{o}}$ ) constitue la face «intérieure» du bifeuillet homonyme (de format $21 \times 15 \mathrm{~cm}$ ), et les folios

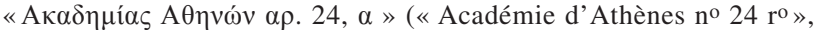

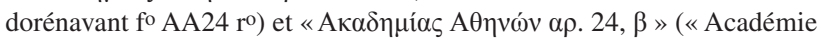
d'Athènes no $24 \mathrm{v}^{\mathrm{o}}$ », dorénavant fo AA24 $\mathrm{v}^{\mathrm{o}}$ ) sont les deux faces du feuillet portant le même nom (de format $28 \times 17 \mathrm{~cm}$ ).

7. Voir AE, vol. I, p. 415, 417-418.

8. Protocole de transcription: La transcription mime l'organisation de la page manuscrite, reproduisant au plus près possible la justification des lignes et les situations des blocs d'écriture les uns par rapport aux autres. L'orthographe, l'accentuation des mots grecs et la ponctuation de Solomos ont été scrupuleusement respectées. Les mots et les phrases raturés sont barrés dans la transcription. Les ajouts interlinéaires sont reproduits à leur place et en caractères plus petits. D'une manière générale, la variation des corps suit les différences observables dans la graphie. Les corrections en surcharge que nous avons pu déchiffrer sont reproduites à leur place, en 
de restituer la spatio-temporalité de l'écriture de Solomos dans chacune des trois pages, ce qui permettra également au lecteur d'étudier en détail le va-et-vient entre l'italien et le grec. Quant aux transcriptions-traductions (voir fig. 2, 5, 8), nous utilisons deux couleurs différentes afin de distinguer les deux langues d'écriture dans les documents originaux (rouge pour le grec, vert pour l'italien), et traduisons tout en français ${ }^{9}$, sauf les segments syllabiques indiquant la rime en fin des mots grecs à venir (par exemple, vı [fig. 5] ; $\varepsilon v \alpha$ [fig. 8]).

\section{La répartition opérationnelle des deux langues}

Malgré l'interaction permanente entre l'italien et le grec dans la genèse de l'œuvre de Solomos, le degré de présence de l'une ou de l'autre langue varie sensiblement d'un manuscrit à l'autre, d'une page à l'autre. Ainsi, dans le fo AA24 ro (fig. 4) c'est l'italien qui domine dans l'écriture, alors qu'au sein du fo AA24 vo (fig. 7) c'est le grec qui joue le rôle primordial dans les opérations génétiques. En revanche, on constate un équilibre relatif dans l'utilisation des deux langues dans la page AA20, $1 v^{o}-2 r^{\circ}$ (fig. 1).

On peut remarquer dans ces trois feuillets une tendance à la séparation fonctionnelle entre les deux langues 10 : pour schématiser, dans la plupart des cas l'italien endosse le rôle du scénarique (le processus consacré au travail de planification, d'organisation et de structuration des unités thématiques-narratives), tandis que le grec assume le rôle du scriptural (le processus de textualisation) ${ }^{11}$. Quant à la langue grecque, dans les trois pages explorées ici, il sert principalement à la composition des vers ou, dans un cas de la page AA20, 1vo-2ro (fig. 1), à la recherche de rimes (voir fig. 3, c.e. 6 du premier quart de la page). En revanche, l'écriture scénarique en italien intervient dans des opérations de genèse très variées : scénarios, esquisses, résumés, notes de régie. Enfin, dans le fo AA24 vo (fig. 7) on peut repérer un autre type d'opération scripturale en langue italienne (voir fig. 9, c.e. 1) : il s'agit d'une citation tirée d'un ouvrage du théosophe allemand Jacob Böhme (1575-1624) dans sa traduction en italien par Nikolaos Lountzis (1798-1885) ${ }^{12}$, suivi d'un commentaire de Solomos (voir fig. 9, c.e. 2). Malgré cette séparation fonctionnelle des deux langues, on remarque de nombreuses interférences entre l'italien et le grec.

\section{"Quand l'autre langue intervient sans être sollicitée $13 »$ : les interférences entre l'italien et le grec}

Les interférences entre l'italien et le grec dans les manuscrits de travail de Solomos sont nombreuses et se manifestent à tous les niveaux : graphématique-phonétique, morphosyntaxique et sémantique ${ }^{14}$. Dans la très grande majorité des cas, c'est l'italien qui interfère sur la langue grecque. Cela pourrait constituer une preuve supplémentaire que la langue dominante de Solomos est l'italien, compte tenu du fait que plusieurs spécialistes éminents du bilinguisme soulignent l'importance de la

caractères plus grands et en italique. Les mots illisibles sont indiqués par l'abréviation [illis.]. Ceux dont la lecture est incertaine ou conjecturale sont encadrés par deux astérisques: *buone* (fig. 6).

9. La traduction des passages en italien est inédite. Une partie non négligeable de la traduction des vers ou des syntagmes grecs est aussi inédite; pour le reste, soit nous suivons la traduction de Levesque (Solomos : introduction, prose et poèmes, op. cit.), soit, dans la plupart des cas, nous nous en inspirons à bien des égards. Par ailleurs, nous nous en voudrions de ne pas signaler les suggestions et les révisions importantes proposées par Henri Tonnet et Emilio Sciarrino, respectivement pour la traduction du grec et de l'italien. Nous tenons à les en remercier chaleureusement.

10. Pour le terme «séparation fonctionnelle» des langues durant la genèse d'une œuvre, voir Olga Anokhina, «Étudier les écrivains plurilingues grâce aux manuscrits », dans Écrire en langues : littératures et plurilinguisme, Olga Anokhina et François Rastier (dir.), Paris, Éditions des archives contemporaines, coll. «Multilinguisme, traduction, création», 2015, p. 31-43.

11. Pour un examen détaillé du couple notionnel scénarique/scriptural, voir Pierre-Marc de Biasi, «Qu'est-ce qu'un brouillon? Le cas Flaubert : essai de typologie fonctionnelle des documents de genèse », dans Pourquoi la critique génétique? Méthodes, théories, Michel Contat et Daniel Ferrer (dir.), Paris, CNRS Éditions, coll. «Textes et Manuscrits», 1998, p. 50-51. 12. Il s'agit de l'ouvrage Von den drei Prinzipien göttlichen Wesens [Des trois principes de l'essence divine], 1618-1619 (l'identification dans Georges Véloudis, Dionysios Solomos. Poésie et poétique romantiques. Les sources allemandes [en grec], Athènes, Gnosi, 1989, p. 103).

13. Nous empruntons cette belle phrase, apte à exprimer de manière exemplaire la quintessence du phénomène de l'interférence linguistique, à François Grosjean, Parler plusieurs langues. Le monde des bilingues, Paris, Albin Michel, 2015, p. 76.

14. Voir à ce titre les analyses ainsi que les exemples - quelques-uns d'entre eux viennent des Libres Assiégés - proposés par Aphrodite Athanasopoulou, «Phénomènes d'interférence linguistique dans l'œuvre de Solomos» (en grec), Mandatophoros, vol. XLI, 1996, p. 5-49; Aphrodite Athanasopoulou, «Problèmes de description et d'interprétation du greco-italien de Solomos» (en grec), Porphyras, vol. XCV-XCVI, 2000, p. 197-232. 


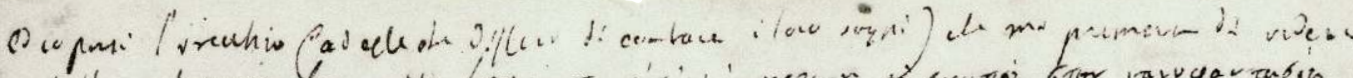

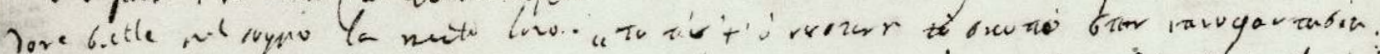

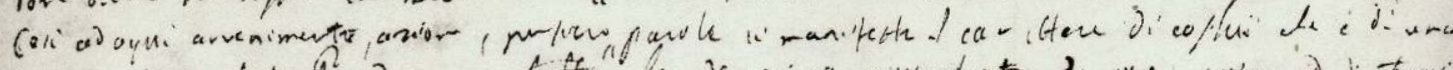

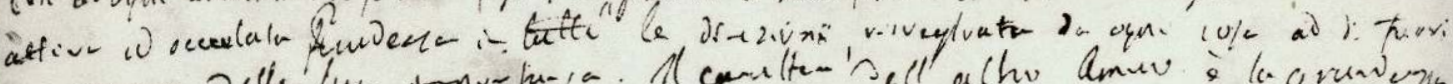

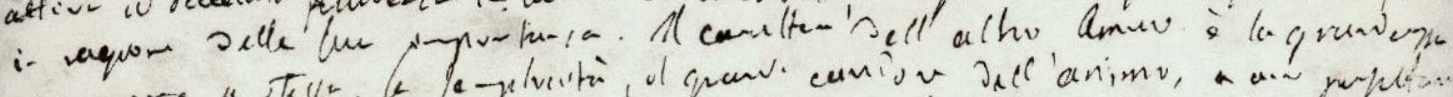

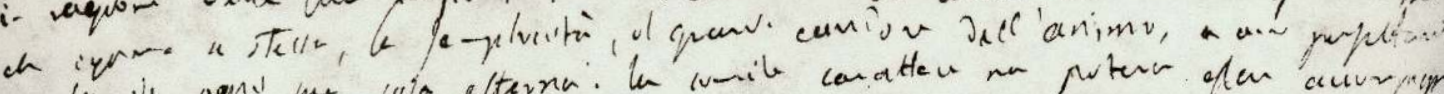

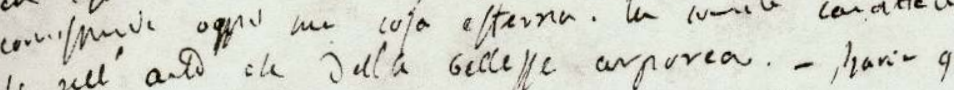

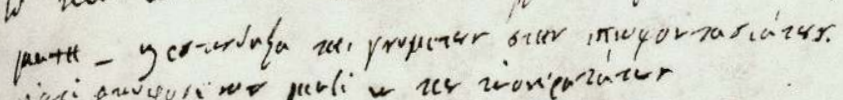

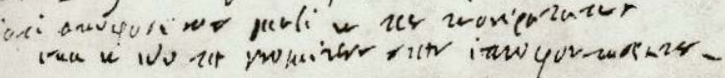

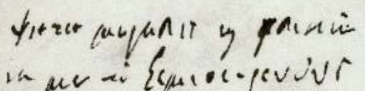

in bata. the

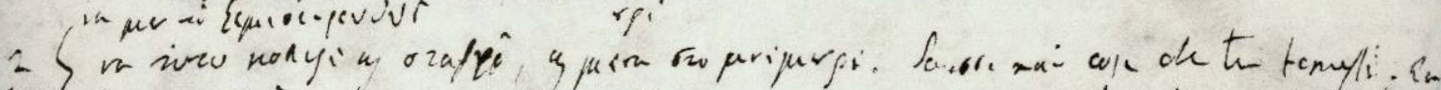

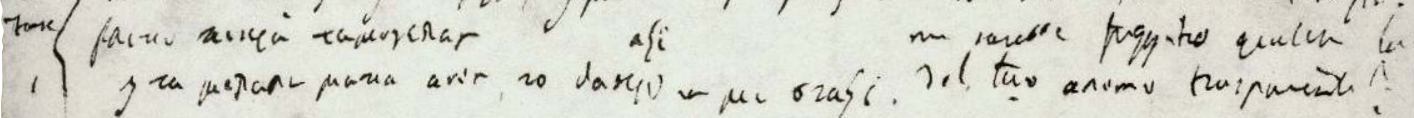

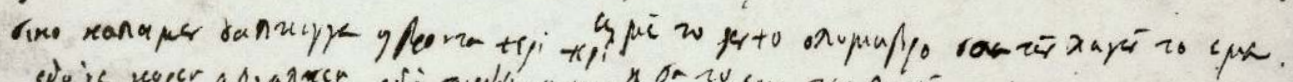

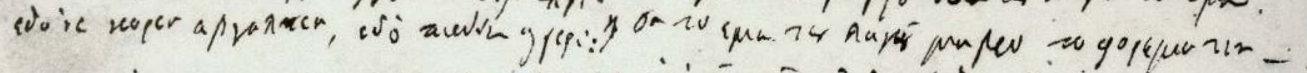

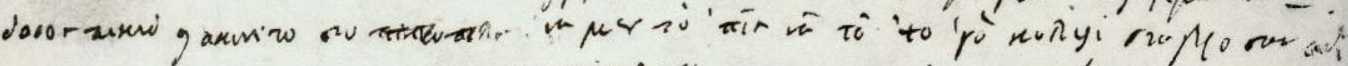

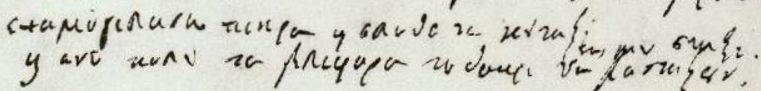

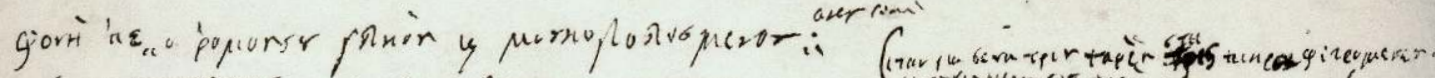

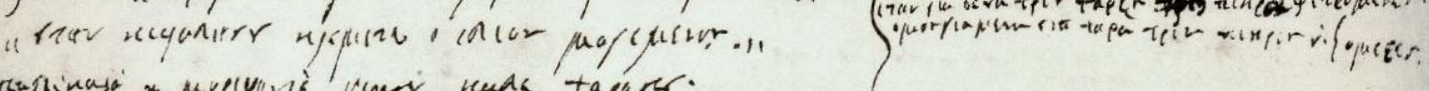

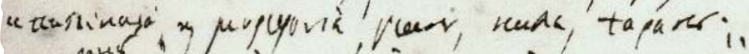

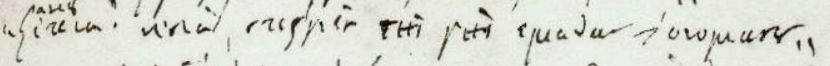

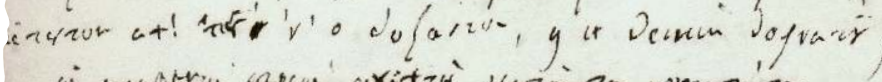

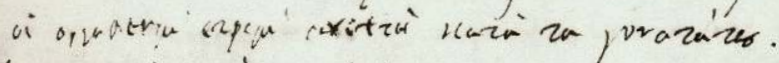

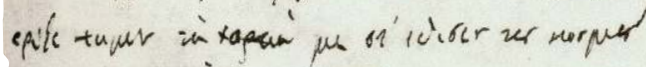

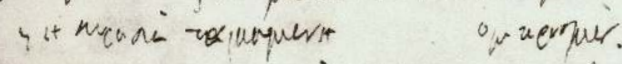

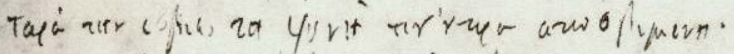

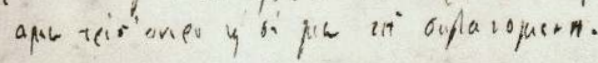

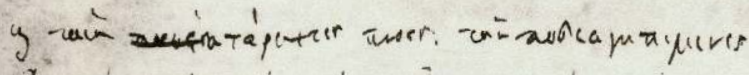

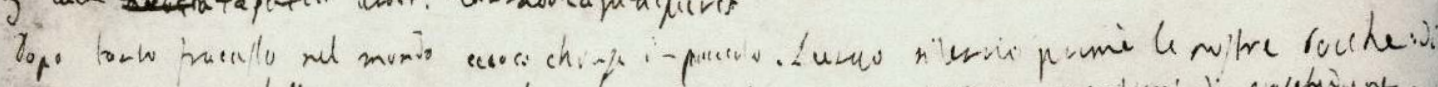

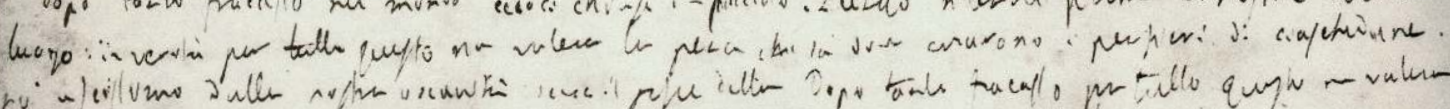

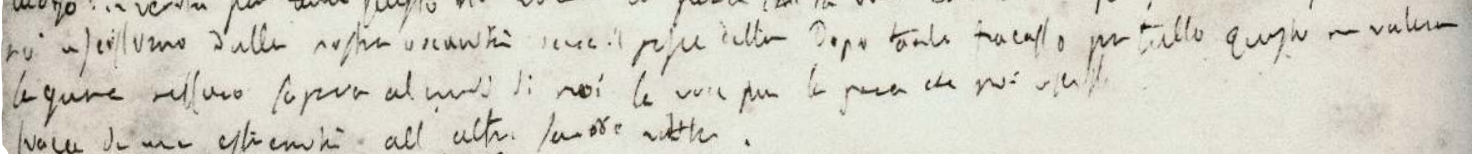

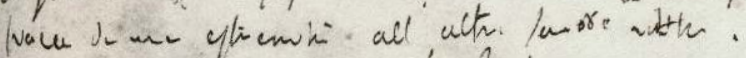

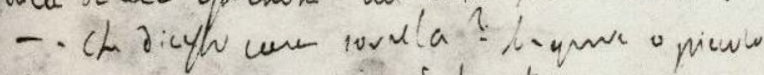

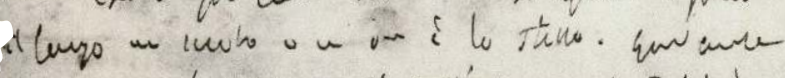

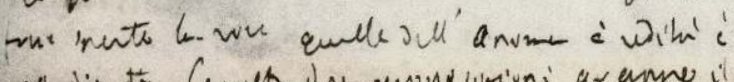

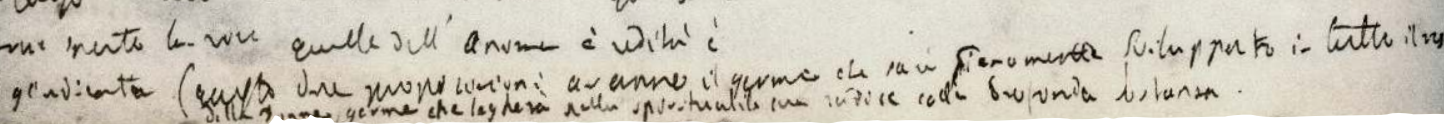




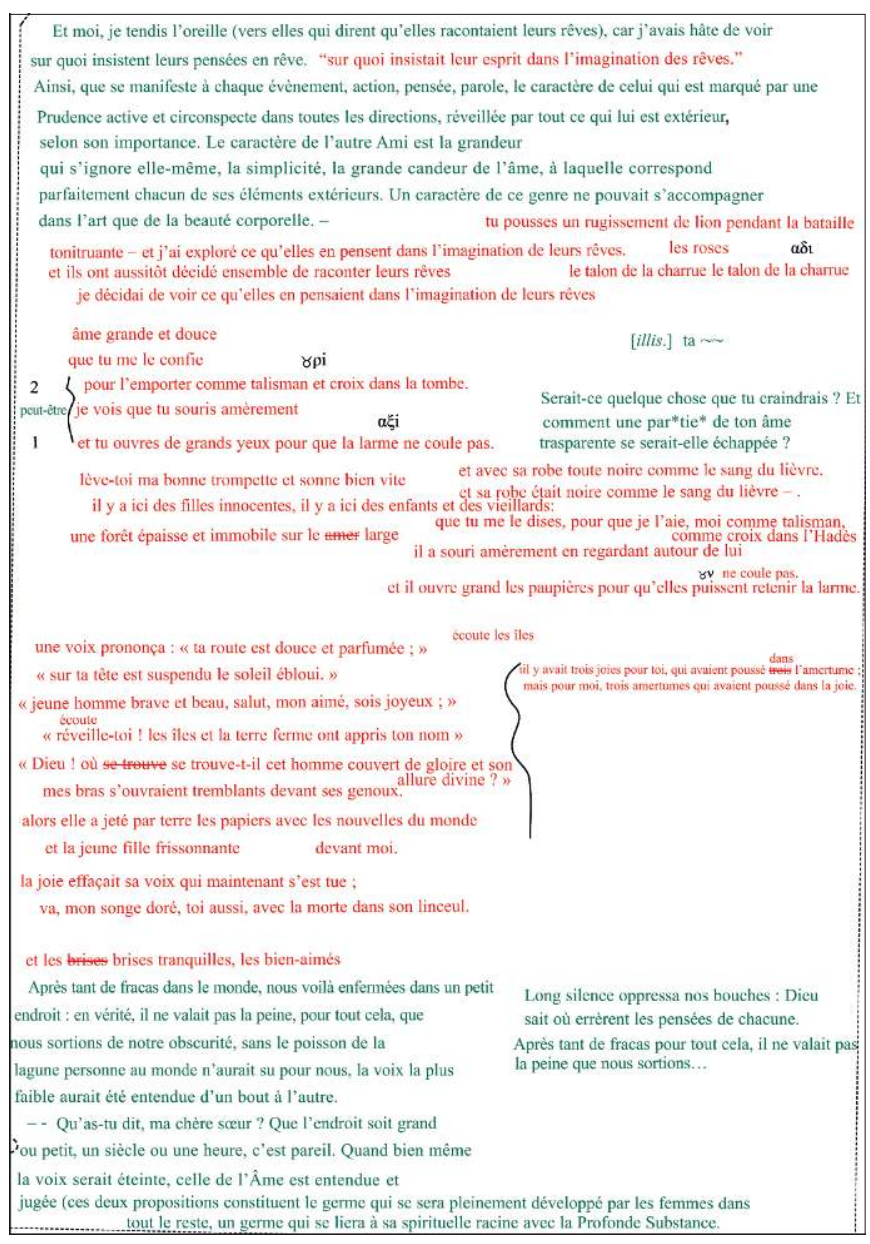

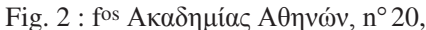

$1 \mathrm{v}^{\mathrm{O}}-2 \mathrm{r}^{\mathrm{O}}\left(\mathrm{AA} 20,1 \mathrm{v}^{\mathrm{O}}-2 \mathrm{r}^{\mathrm{O}}\right)$ : transcription-traduction

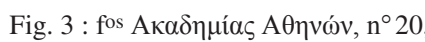
$1 \mathrm{v}^{\mathrm{o}-2} 2 \mathrm{r}^{\mathrm{o}}\left(\mathrm{AA} 20,1 \mathrm{v}^{\mathrm{o}}-2 \mathrm{r}^{\circ}\right)$ : reconstitution des campagnes d'écriture
Ed io porsi I'or chio (ad esse che dissero di contare i loro sogni) che mi premeva di vedere
ouve batte nel Così ad ogni avvenimento azione, pensiero, parola si manifesti il carattere di costui che e di una attiva ed occulata Prude a in tutte le direzionf, risvegliata da ogni cosa al di fuori, in ragione della sua mportanza. II carattere dell'altro Amico è la grandezza che ignora se stessa, la semplicita, il rande candore dell'animo, a cui perfettamente corrisponde 6ghi sua cosa esterna. U 4 imile carattere non poteva essere accompagna-

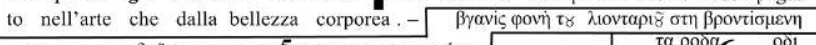

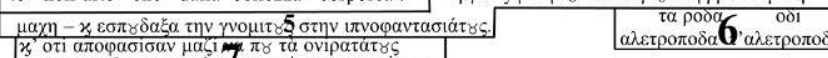

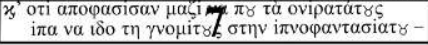

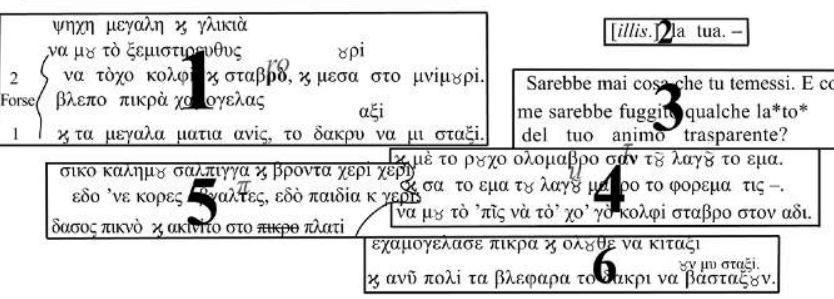

\begin{tabular}{|c|c|}
\hline 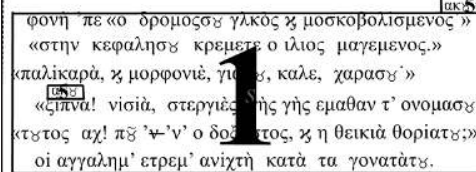 & 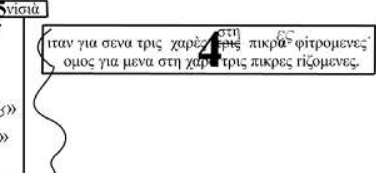 \\
\hline 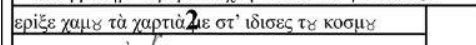 & \\
\hline 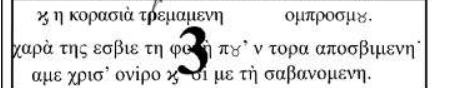 & \\
\hline 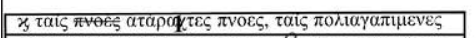 & \\
\hline 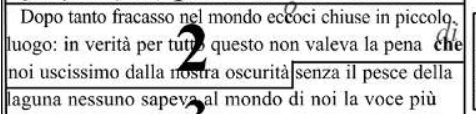 & 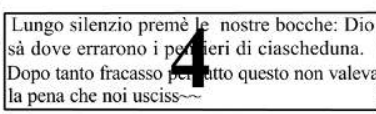 \\
\hline 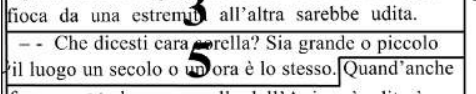 & \\
\hline 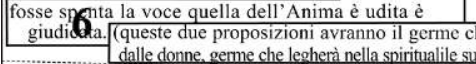 & estc \\
\hline
\end{tabular}




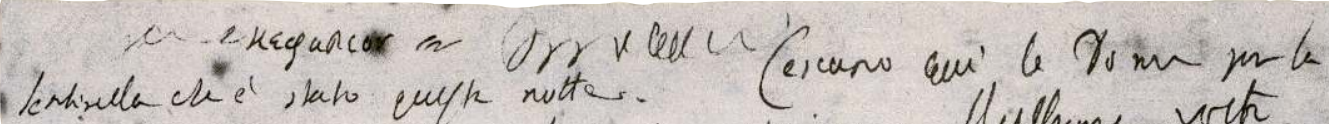

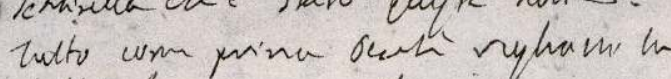

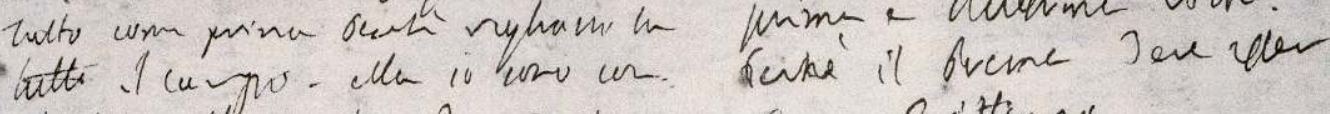

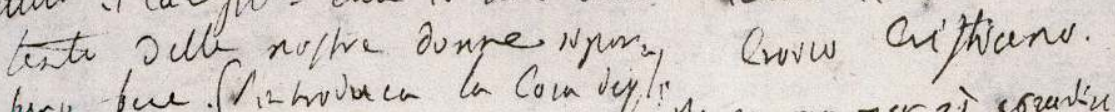

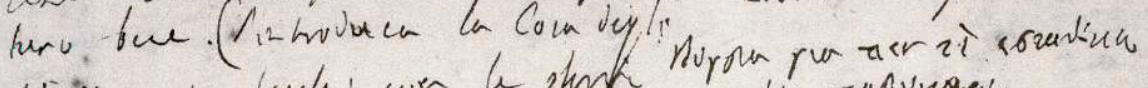

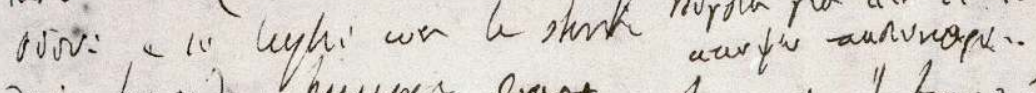

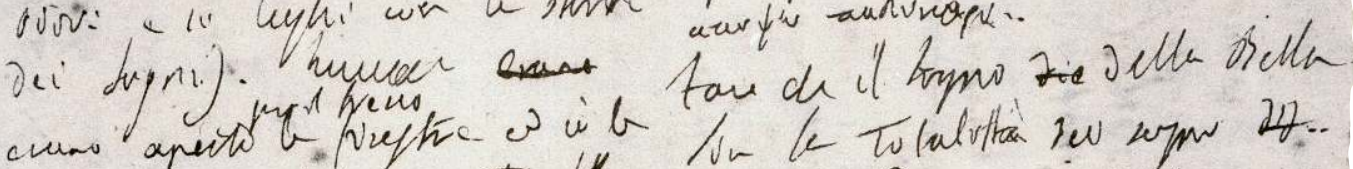
cuaro aperto

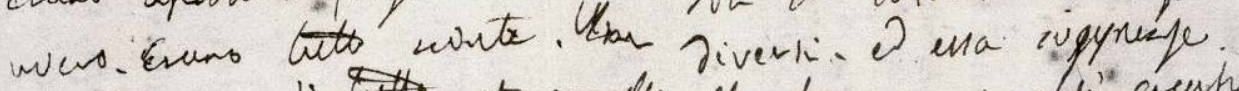

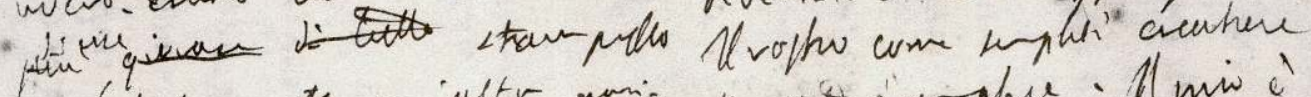

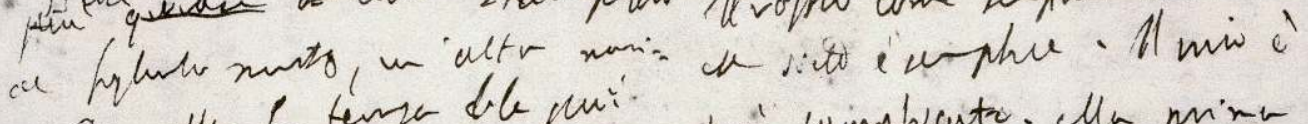

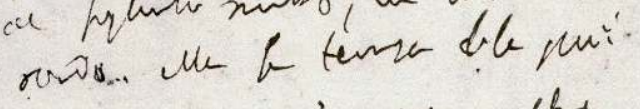

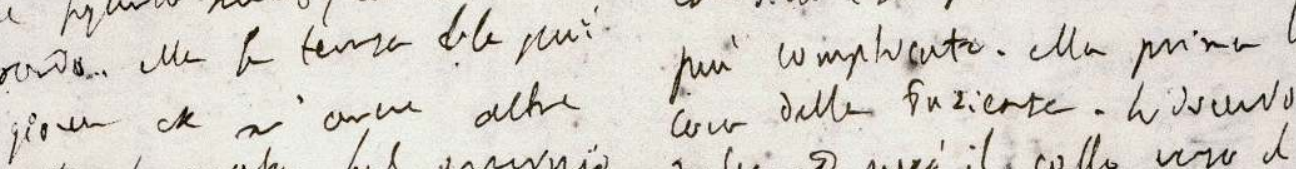

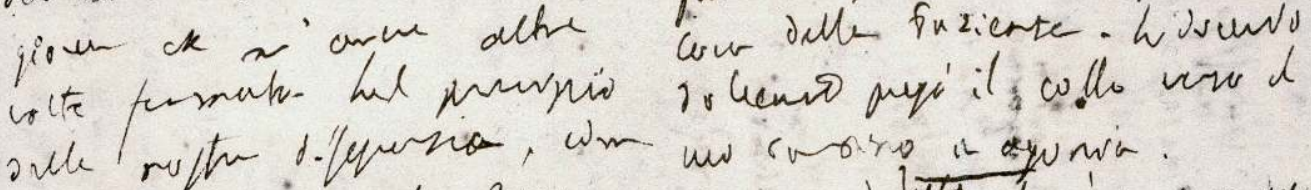

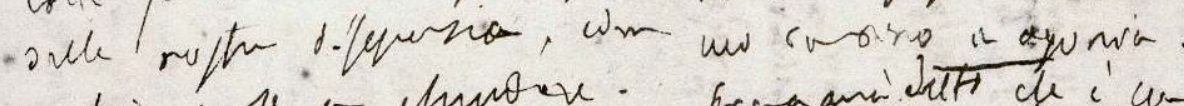

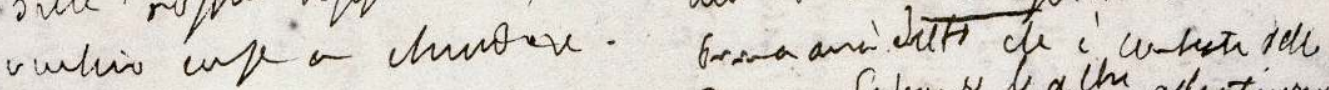

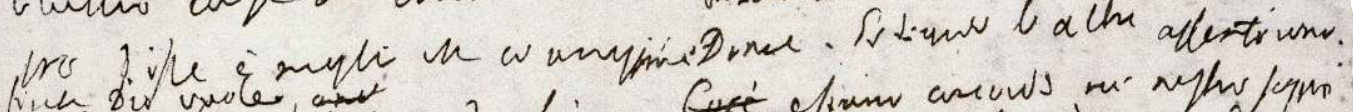

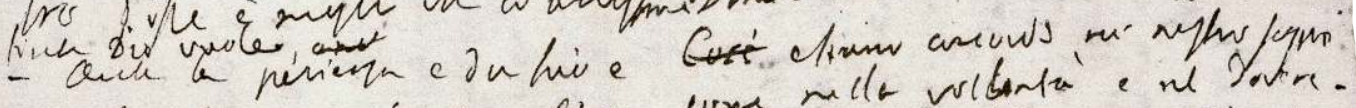

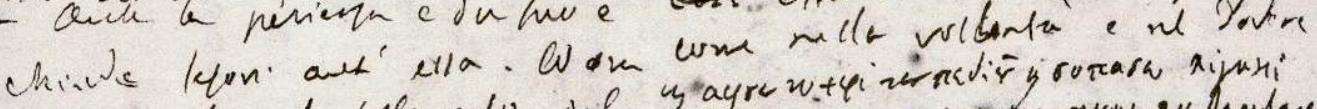

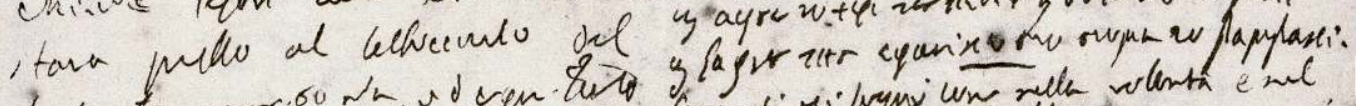

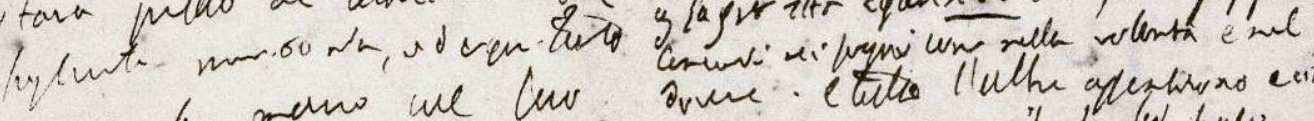

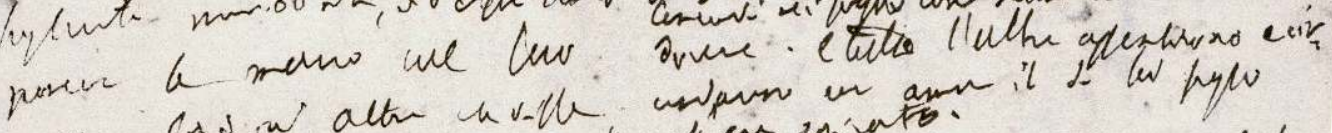

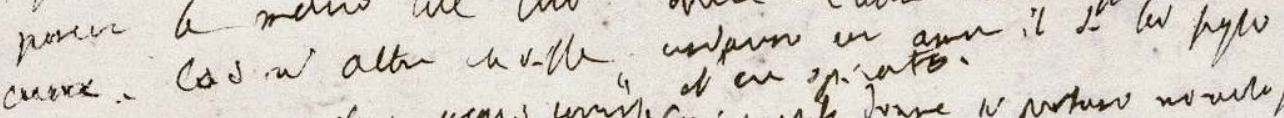

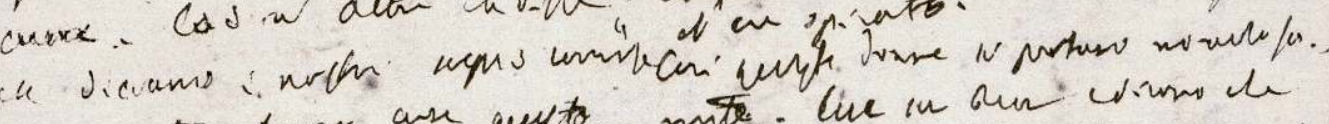

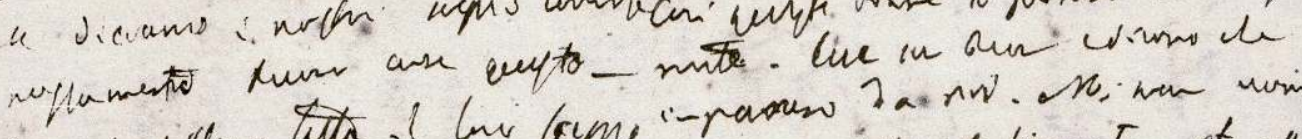

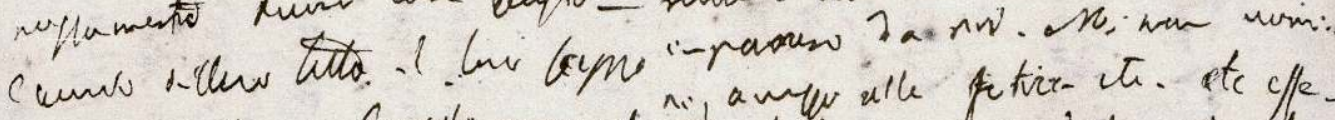

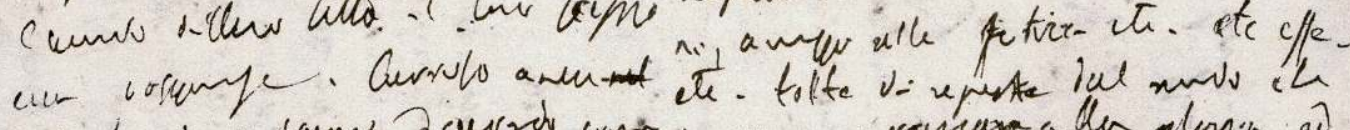

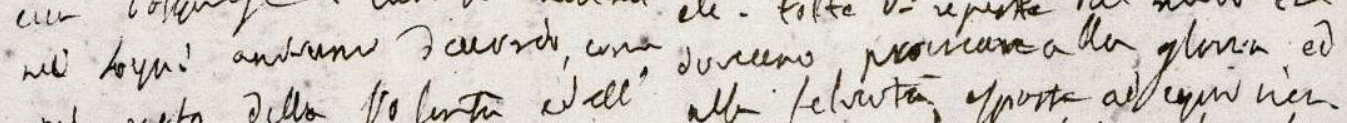

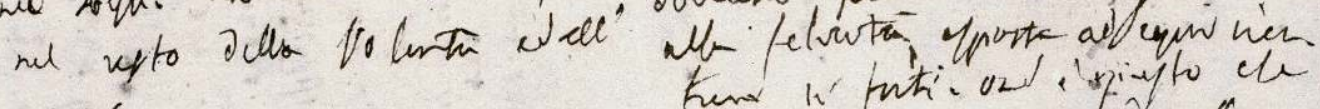
oproake.

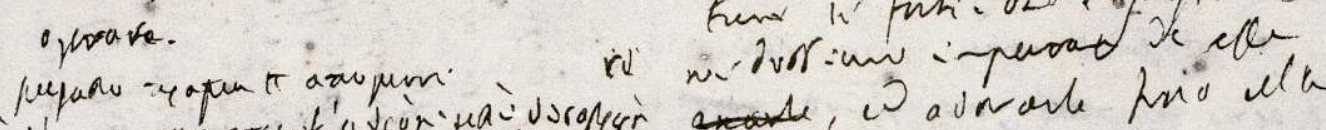

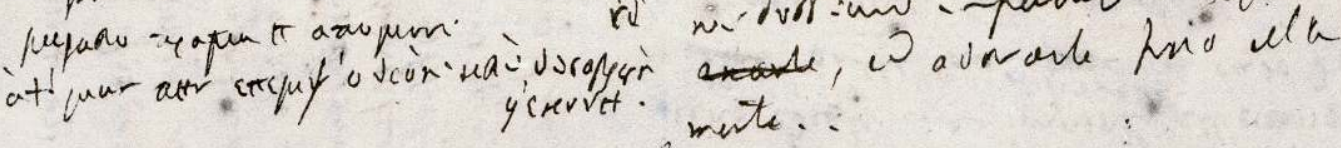

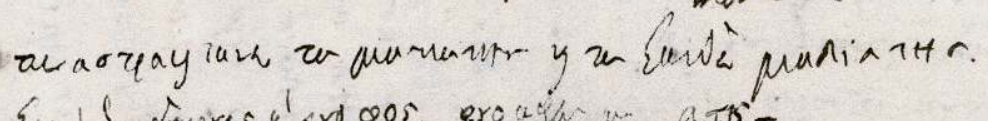

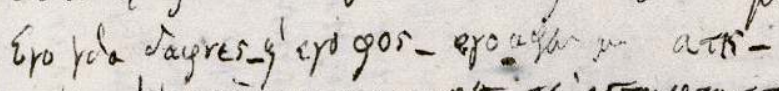

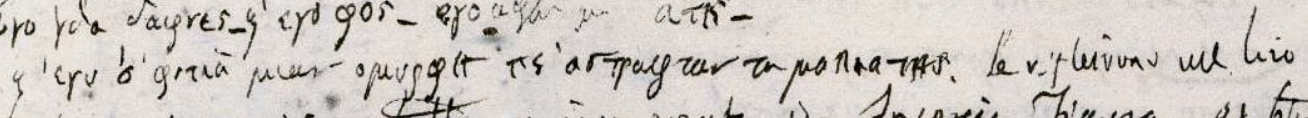

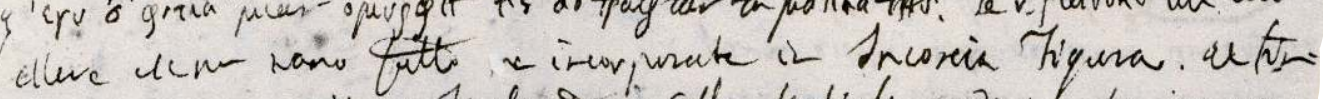

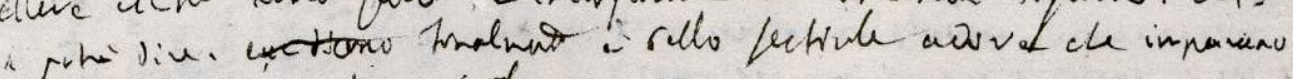
Danvi. Wh allinuntio our eto. 


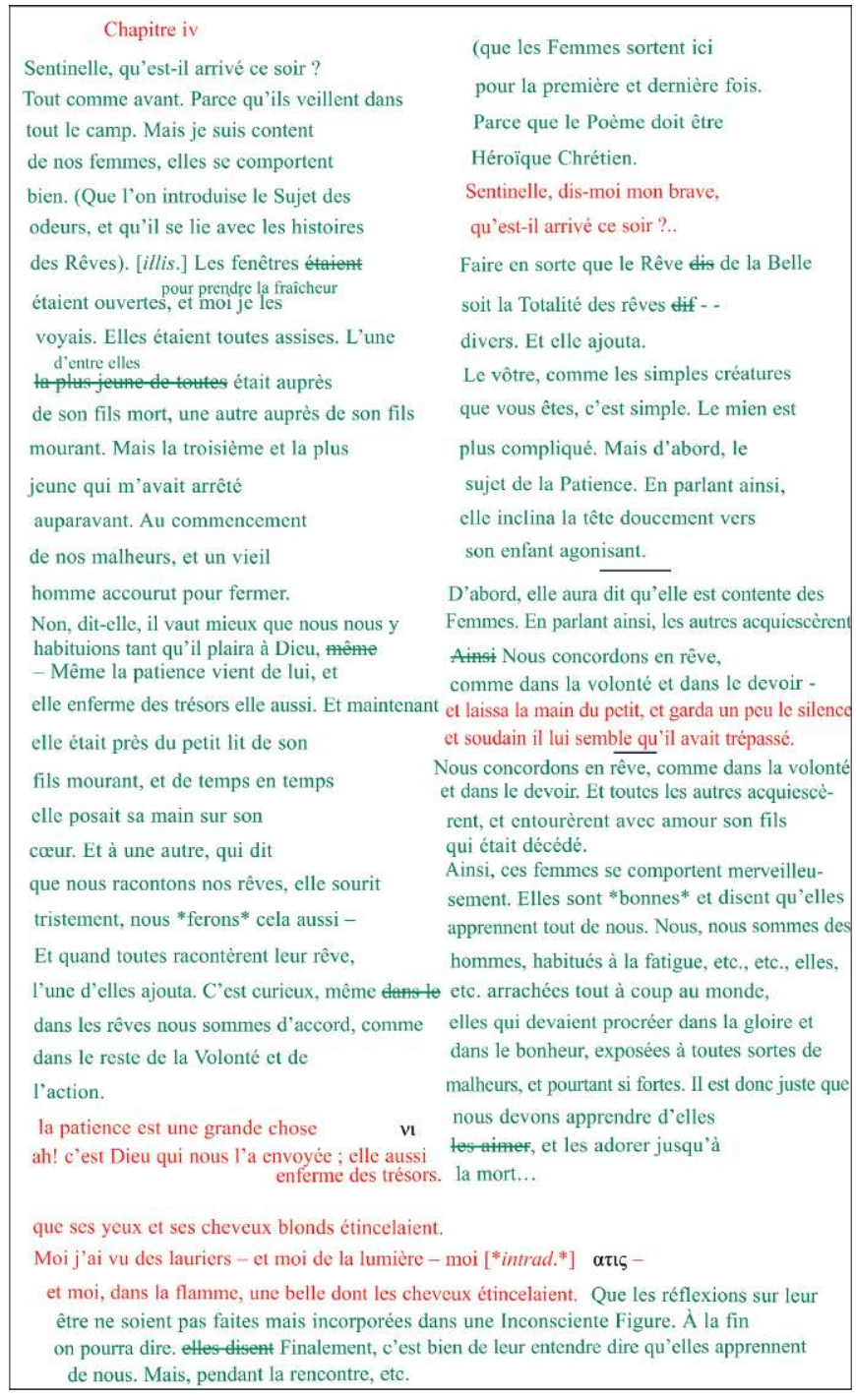

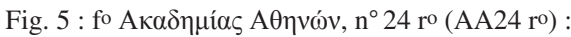
transcription-traduction

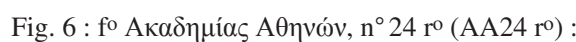
reconstitution des campagnes d'écriture

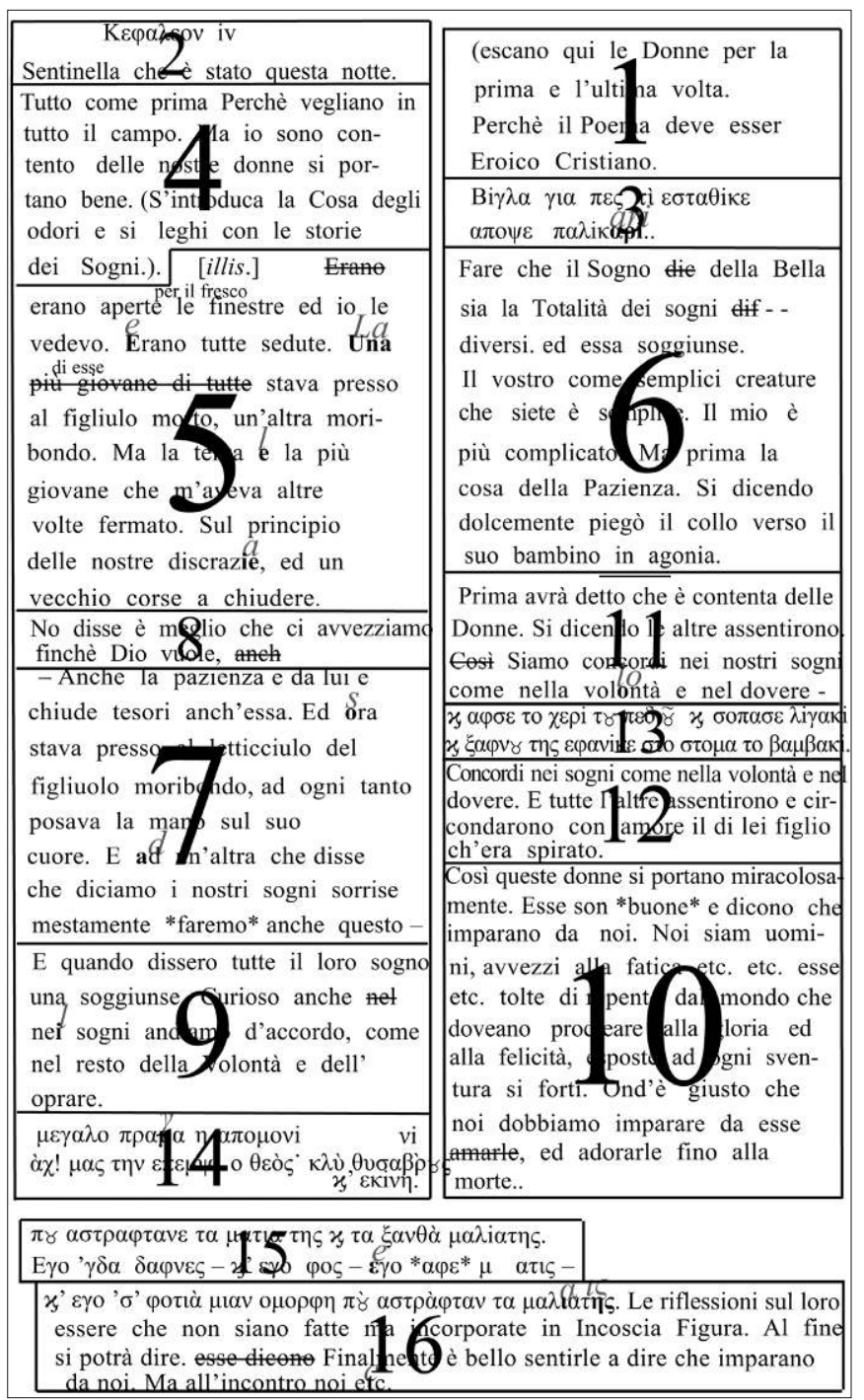




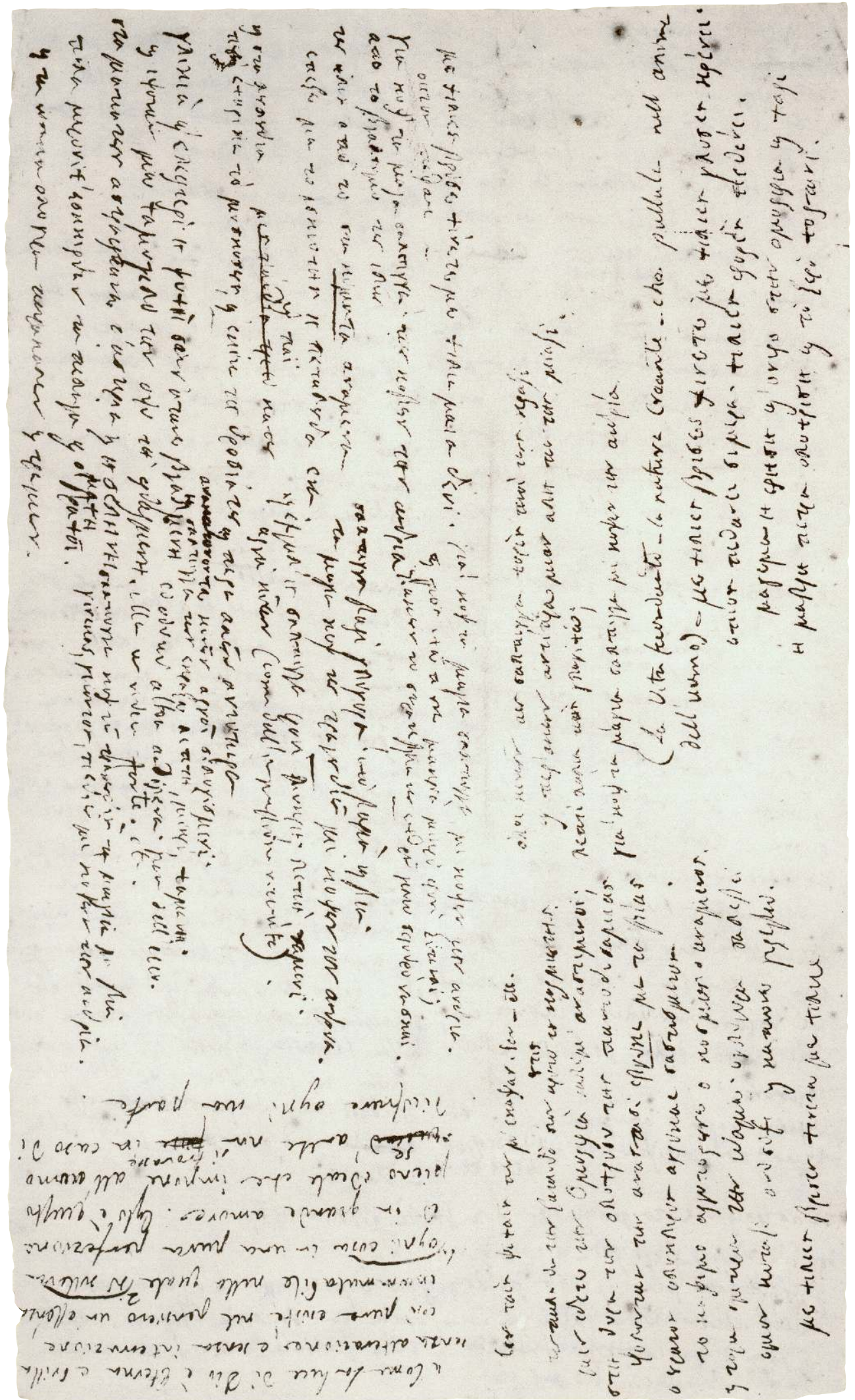



direction des interférences pour déterminer la langue dominante 15 .

Nous nous concentrerons surtout sur le niveau graphématique-phonétique, dont l'évidence dans les trois pages étudiées est de loin la plus forte. Ce type d'interférence est constitué principalement par l'emploi des caractères de l'alphabet latin au sein de mots grecs. On remarquera d'emblée l'utilisation fréquente du caractère latin $i$ au lieu du caractère grec $\imath$ en raison de la similitude visuelle et phonétique qui existe entre les deux lettres (par exemple, $\varepsilon \rho i \xi \varepsilon$ [fig. 1], $\chi \varepsilon \rho i$ [fig. 4], $\chi \imath \lambda i \varepsilon \varsigma$ [fig. 7]).

$$
\begin{aligned}
& \text { spik } \varepsilon \rho i \xi \varepsilon \\
& \text { Fos AA20, 1vo-2ro (fig. 1, détail) } \\
& \chi \varepsilon \rho \mathrm{i} \\
& \text { Fo AA24 ro (fig. 4, détail) } \\
& \text { tian } \chi 1 \lambda \text { ies } \\
& \text { Fo AA24 vo (fig. 7, détail) }
\end{aligned}
$$

Compte tenu du non-respect (voire de l'ignorance) par Solomos des règles et des conventions de l'orthographe grecque, on observe fréquemment également l'utilisation du $i$ latin à la place de tout autre [i] en grec (par exemple, $\mu v i \mu$ ov $i$ [fig. 1], $\sigma \tau \alpha \xi i$ [fig. 1], $\kappa i \tau \alpha \xi i$ [fig. 1], $\pi \mathrm{o} \lambda i$ [fig. 1],

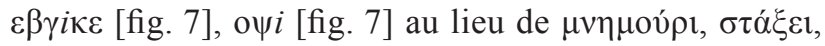

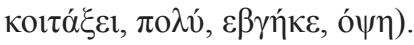

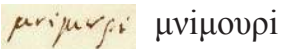

$$
\begin{aligned}
& \text { Fos AA20, 1vo-2ro (fig. 1, détail) } \\
& \operatorname{sen} \xi_{2} \sigma \tau \alpha \xi \mathrm{i} \\
& \text { Fos AA20, 1vo-2ro (fig. 1, détail) } \\
& \mu \sqrt{2}\}: \kappa i \tau \alpha \xi \mathrm{i} \\
& \text { Fos AA20, 1vo-2ro (fig. 1, détail) } \\
& \text { หrคv } \pi \mathrm{O} \lambda \mathrm{i} \\
& \text { Fos AA20, 1vo-2ro (fig. 1, détail) }
\end{aligned}
$$

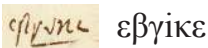

$$
\begin{aligned}
& \text { Fo AA24 vo (fig. 7, détail) } \\
& \checkmark \text { } \checkmark \psi \text { i } \\
& \text { Fo AA24 vo (fig. 7, détail) }
\end{aligned}
$$

Par ailleurs, la parfaite ressemblance phonétique entre, d'un côté, les graphèmes $\langle\mathrm{j} \alpha\rangle$ et $\langle\gamma i \alpha\rangle$ (tous les deux prononcés [ja]) et, d'un autre côté, entre les graphèmes $<$ ro $>$ et $<\rho o>$ (l'un et l'autre articulés [ro]) et les graphèmes $<$ ri $>$ et $\langle\rho \mathrm{l}\rangle$ (tous les deux prononcés [ri]) amène Solomos à écrire respectivement $\mu \alpha j \alpha$ (fig. 7), $\gamma \varepsilon r o v \tau o \varsigma$ (fig. 7)

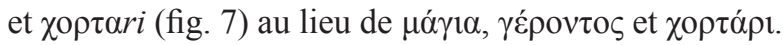

$$
\begin{aligned}
& \mu \ldots j_{*} \mu \alpha \alpha \alpha \\
& \text { Fo AA24 vo (fig. 7, détail) } \\
& \text { rivingr yerovios } \\
& \text { Fo AA24 vo (fig. 7, détail) }
\end{aligned}
$$

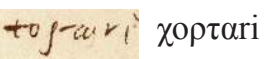

$$
\begin{aligned}
& \text { Fo AA24 vo (fig. 7, détail) }
\end{aligned}
$$

Dans certains cas, Solomos se rend compte de ses erreurs en les corrigeant immédiatement, ce dont témoignent les modifications en surcharge 16 :

$$
\begin{aligned}
& \sigma z a / \hat{\varphi}_{i}, \sigma \tau \alpha \beta \rho \grave{\rho} / r \\
& \text { Fos AA20, 1vo-2ro (fig. 1, détail) } \\
& \text { Aujé } \lambda \alpha \gamma \mathbf{0} / u \\
& \text { Fos AA20, 1vo-2ro (fig. 1, détail) } \\
& \text { zQilupul/t } \tau \rho \varepsilon \mu \alpha \mu \varepsilon v \eta / r \\
& \text { Fos AA20, 1vo-2ro (fig. 1, détail) }
\end{aligned}
$$

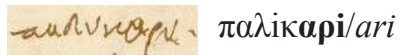

$$
\begin{aligned}
& \text { Fo AA24 ro (fig. 4, détail) } \\
& \text { Qjo } \mathrm{\varepsilon} \gamma \mathrm{O} / \mathrm{e} \\
& \text { Fo AA24 ro (fig. 4, détail) }
\end{aligned}
$$

Ces interférences «éphémères » (c'est-à-dire surgies et corrigées au fur et à mesure de l'écriture) entre les systèmes

\footnotetext{
15. Voir, par exemple, François Grosjean, op. cit., p. 80-81 : « La direction des interférences dépend quant à elle en partie de la dominance que l'on possède des langues. Une langue fortement dominante viendra influencer la ou les langues les plus faibles.»

16. Les corrections en surcharge sont indiquées par l'emploi du caractère gras et les lettres surchargées sont placées à la fin du mot, après la barre oblique et en italique.
} 
graphiques latin et grec ne peuvent s'interpréter, une fois de plus, que par la forte parenté des graphèmes au niveau phonétique : $\langle\mathrm{r}\rangle$ et $\langle\rho\rangle=[\mathrm{r}] ;\langle\mathrm{u}\rangle$ et $\langle\mathrm{ov}\rangle=[\mathrm{u}] ;<$ ari $>$ et $\langle\alpha \rho \mathrm{l}\rangle=[$ ari] $;<\mathrm{e}>$ et $\langle\varepsilon\rangle=[\mathrm{e}]$.

Enfin, le syntagme « ki $\mu \alpha \tau \alpha \alpha v \alpha \mu \varepsilon v \alpha »$ [vagues allumées] (fig. 7) constitue un autre bon exemple d'interférence possible, cette fois au niveau syntaxique :

$$
\begin{aligned}
& \text { H. } \\
& \text { Fo AA24 vo (fig. 7, détail) }
\end{aligned}
$$

Plus précisément, la postposition de l'adjectif épithète, qui est situé après le nom dans ce syntagme ( «i $\kappa \alpha \tau \alpha$ $\alpha v \alpha \mu \varepsilon v \alpha »$ [vagues allumées]) peut être considérée comme le résultat de l'influence de la syntaxe de la langue italienne sur celle de la langue grecque; en effet, alors que la postposition de l'adjectif épithète est fréquente en italien, elle est très inhabituelle dans la langue grecque moderne, où, normalement, l'adjectif est placé avant le nom (la forme attendue en grec serait donc « $\alpha v \alpha \mu \varepsilon v \alpha$ кi $\mu \alpha \tau \alpha »$ [allumées vagues]) ${ }^{17}$.

\section{Le code-switching}

Outre la première pratique évoquée plus haut, qui sert à mettre en place une séparation fonctionnelle des deux langues, l'observation des trois pages présentées ici permet de repérer une pratique d'écriture diamétralement opposée : le code-switching. Selon la définition établie par les linguistes, cette pratique réside dans l'alternance de deux ou de plusieurs codes linguistiques au sein du même énoncé. Il s'agit d'une pratique assez typique de l'écriture solomienne18 :

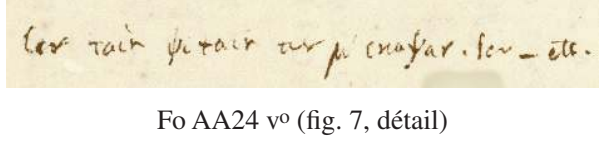

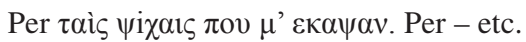

[Pour les âmes qui m'ont brûlé. Pour - etc.] ${ }^{19}$

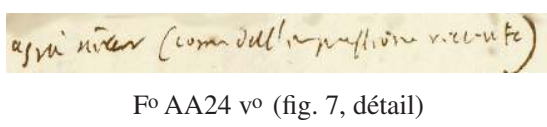

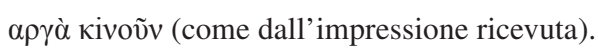

[ils bougent lentement (comme résultat de l'impression reçue).]

$$
\begin{aligned}
& \text { co vivur altow a.dijera pur dell elev. } \\
& \text { Fo AA24 vo (fig. 7, détail) }
\end{aligned}
$$

ed odono altra andipera' pari dell'ecco.

[et ils entendent une autre de l'autre côté; pareil à l'écho.]

Commençons par le premier exemple : la langue de base étant le grec, on remarque l'intrusion de la préposition italienne «per» (pour) au lieu de la préposition correspondante grecque $\gamma 1 \alpha^{20}$. Dans le deuxième exemple, la langue de base est également le grec, auquel s'ajoutent des éléments de la langue italienne sous forme de commentaire entre parenthèses. Pourtant, ce commentaire fonctionne en même temps comme une esquisse, dont l' «exécution» grecque va compléter le vers à un stade ultérieur :

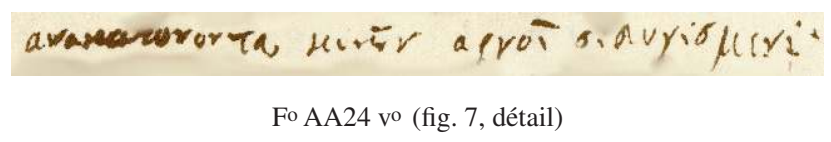

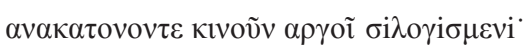

[ils se remuent, ils bougent lents et pensifs ;]

Dans le troisième exemple, la langue de base est l'italien, dans laquelle intervient un mot grec («andipera» qui est une romanisation de l'adverbe grec $\alpha v \tau i ́ \pi \varepsilon \rho \alpha$, qui signifie «de l'autre côté»).

Cette esquisse en langue mixte (italien-grec) présente également des caractéristiques rythmiques intéressantes. C'est sur ce dernier aspect que nous allons nous pencher à présent.

\section{La prose rythmique et la transmétrisation avant-textuelle}

$\mathrm{Si}$, comme on vient de le voir, la séparation des langues est loin d'être respectée par le poète, de même la frontière

17. Pour plus de détails sur ce sujet, voir Aphrodite Athanasopoulou, «Phénomènes d'interférence linguistique», op. cit., p. 14-16.

18. Pour une première approche de ce sujet, voir Aphrodite Athanasopoulou, «Problèmes de description», op. cit., p. 213-216.

19. Ici et plus loin, dans la traduction française des énoncés cités, les mots ou les syntagmes grecs figurent en italique.

20. Dans ce contexte, l'utilisation de l'adverbe «etc. » est considérée comme négligeable, compte tenu de sa fonction métascripturale. 
entre la prose et la poésie est parfois peu étanche chez Solomos, car cette même «prose» peut avoir une forme rythmique ou versifiée. Ce phénomène est particulièrement manifeste dans l'avant-texte de Lambros, l'œuvre grecque de Solomos composée en octaves italiennes d'hendécasyllabes (endecasillabi) ${ }^{21}$. En fait, d'après une lecture métrique des manuscrits, ce même phénomène est présent, bien que dans une moindre mesure, dans le premier projet des Libres Assiégés, même si celui-ci n'est pas écrit en hendécasyllabes mais en décapentasyllabes (le vers typique de la poésie grecque, rappelons-le). Nous présenterons ici deux exemples d'hendécasyllabes bien «dissimulés 22 » dans les esquisses venant du fo AA24 ro (fig. 4) :

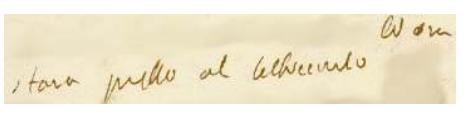

Fo AA24 ro (fig. 4, détail)

Ed ora stava presso al letticciulo

[Et maintenant elle était près du petit lit]

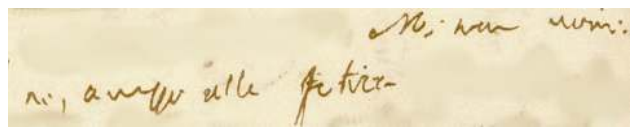

Fo AA24 ro (fig. 4, détail)

Noi siam uomini, avvezzi alla fatica

[Nous, nous sommes des hommes, habitués à la fatigue]

L'identification des deux hendécasyllabes italiens cidessus ne nous surprend pas, ni sur le plan génétique (faisant partie des esquisses, il paraît normal que les deux énoncés soient centrés sur un premier essai de textualisation en langue italienne, afin que le passage du poète bilingue à la rédaction de vers en grec soit facilité par le rythme de l'hendécasyllabe), ni sur le plan syntaxique et rhétorique (début de la période).

Regardons à présent deux autres exemples :

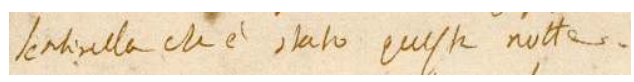

Fo AA24 ro (fig. 4, détail)

Sentinella che è stato questa notte.

[Sentinelle, qu'est-il arrivé ce soir?]

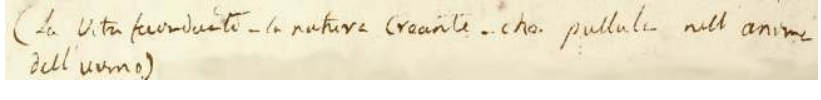

Fo AA24 vo (fig. 7, détail)

(La Vita fecondante - la natura Creante - che pullula nell anima dell'uomo)

([La vie fécondante - la nature Créante - qui pullule dans l'âme de l'homme])

Il s'agit dans le premier énoncé d'un cas d'hendécasyllabe, et sa disposition autonome sur la page permet son identification. Le deuxième cas constitue un très bon exemple de cohabitation entre deux heptasyllabes (settenari) et un hendécasyllabe. La coexistence de ces deux types de vers n'est par ailleurs pas étrangère ni à la tradition métrique italienne ni à la production en langue italienne de Solomos ${ }^{23}$.

21. Voir Massimo Peri, «Petites remarques sur Solomos» (en grec), Mandatophoros, vol. 41, 1996, p. 83-95; Massimo Peri, «L'italiano di Solomòs », dans Scrittori stranieri in lingua italiana dal Cinquecento ad oggi, Atti del Convegno Internazionale di Studi (Padova, 20-21 marzo 2009), Furio Brugnolo (dir.), Padoue, Unipress, 2009, p. 285-287. Il faut rappeler à cet égard que Solomos est extrêmement habitué aux schémas rythmico-syntaxiques de l'hendécasyllabe italien, au point que la composition de ce vers est presque «automatique» chez lui ; cela vient principalement de la longue expérience du poète dans l'exécution improvisée d'hendécasyllabes sur des rimes données, dont le résultat a été, parmi d'autres choses, son recueil de poèmes en italien Rimes improvisées (Rime improvvisate, 1822).

22. Comme le dit bien Benoît de Cornulier, Art poëtique. Notions et problèmes de métrique, Lyon, Presses universitaires de Lyon, 1995, p. 21 :

«Ce qui est métrique n'est donc pas un vers, mais des vers, par équivalence mutuelle; et ce qu'on appelle le mètre d'un vers singulier consiste moins en la conformité de cette expression singulière avec une norme abstraite qu'en un rapport réciproque d'équivalence contextuelle en nombre syllabique entre plusieurs suites verbales voisines disjointes. » Il s'agit, bien entendu, des conditions pratiquement inexistantes dans la succession désordonnée des énoncés du scénarique en italien chez Solomos. Par ailleurs, un découpage arbitraire de ces énoncés peut donner lieu à une lecture métrique également arbitraire, apte à reconnaître partout dans le matériau scénarique des entités métriques. Bien qu'il existe quelques «sièges privilégiées" dans la phrase où l'intention métrique est plus évidente, comme par exemple le début et surtout la fin de la période, il faut constamment en faire une lecture ouverte et une analyse au cas par cas. 23. Voir, par exemple, l'Ode pour la première messe (Ode per prima messa, entre 1815-1818), dans Dionysios Solomos, Euvres complètes, vol. II : Prose grecque et æuvres italiennes (introduction, notes et commentaires en grec), éd. par Linos Politis, Athènes, Icaros, 1968, p. 89-92. 
En outre, la séparation des énoncés par un tiret, qui souligne l'autonomie syntaxique et sémantique de chacun d'entre eux, et l'existence d'une rime riche (fecondante - creante) constituent des indices supplémentaires du fait qu'il s'agit vraiment d'une série de trois vers italiens. Enfin, dans les deux cas, les vers constituent des esquisses des décapentasyllabes à venir, comme si la versification italienne était indispensable pour que le poète procède ensuite à la composition de vers en grec.

Cependant, la situation ne peut que se compliquer davantage si nous regardons de plus près le cas suivant :

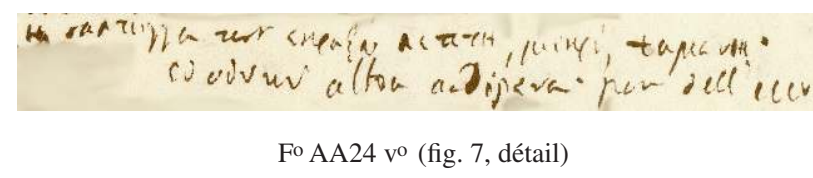

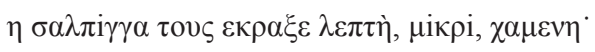
ed odono altra andipera' pari dell'ecco.

[la trompette, faible, ténue et lointaine, les a appelés; et ils entendent une autre de l'autre côté; pareil à l'écho.]

Le deuxième énoncé, en plus d'être un bon exemple de code-switching, constitue, à double titre, un petit paradoxe dans l'univers solomien. En ce qui concerne tout d'abord son statut génétique, il semble qu'il représente un de ces rares cas où Solomos tente de transposer en italien, dans une forme condensée, un vers grec protéiforme attesté dans un large éventail de variantes 24 . Voici le vers grec le plus proche de l'énoncé en italien au niveau lexico-syntaxique, venant de la phase originelle du projet :

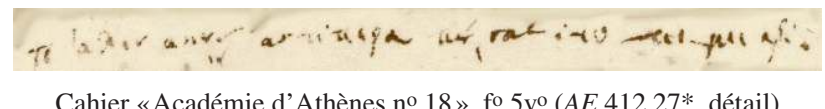

Cahier «Académie d'Athènes no 18 », fo 5vo (AE 412 27*, détail $)$

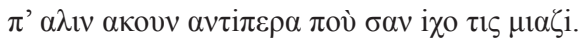

[qui en entendent une autre, de l'autre côté, qui lui ressemble comme un écho.]

Il semble pourtant que le motif de cette traductionadaptation en langue italienne est surtout métrique; d'où la deuxième situation paradoxale, car cet énoncé en italien est loin de constituer un vers italien connu, c'est-à-dire codifié ou documenté dans la tradition métrique italienne. Et pourtant, en suivant de près l'analyse de Massimo Peri à propos d'un cas similaire attesté dans un brouillon du Crétois $^{25}$, Solomos construirait un «décatrisyllabe» en italien (un vers de treize syllabes, inexistant dans la tradition métrique italienne), qui présente une structure analogue au décapentasyllabe grec qui le précède : le premier hémistiche (« ed odono altra andipera 26 ») est identique au premier hémistiche du décapentasyllabe; le deuxième hémistiche présente un pentasyllabe («pari dell'ecco») au lieu de l'heptasyllabe.

Comme le fait remarquer Peri 27 , dans ces cas on pourrait parler de transmétrisation, pour reprendre librement un terme célèbre de Gérard Genette 28 . Alors que la transmétrisation chez Genette désigne la transposition métrique d'un texte à un autre, Peri élargit le sens du terme, qui pour lui se réfère également à «la gestation d'un seul texte ${ }^{29}$ ». Or, cette «gestation» est avant-textuelle, puisque c'est dans des documents de genèse qu'elle se déroule. On pourrait donc même parler de transmétrisation avant-textuelle, propre à l'écriture d'un scripteur bilingue par excellence.

\section{Conclusion}

L'analyse de ces trois pages manuscrites nous amène à la conclusion que, dans l'écriture de Solomos, l'italien n'a pas uniquement le rôle d'une métalangue qui remplirait la fonction scénarique préparant la textualisation en grec à venir, comme le présentent fréquemment les études

24. Cet éventail trouve ses origines dans un petit cahier (de cinq feuilles à peine), qui constitue le document principal dans lequel s'amorce la première phase rédactionnelle du projet, et il se termine dans le manuscrit étudié, c'est-à-dire dans le fo AA24 vo (voir fig. 9, c.e. 7 et 9). Il s'agit,

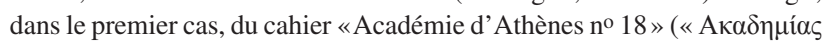
A 0 ๆ

25. Voir Massimo Peri, La «femme vêtue de lune» de Solomos. Une inconnue qui doit rester inconnue (en grec), trad. de l'italien par Kostis Pavlou, Athènes, Gutenberg, 2016, p. 24-25.

26. Dans ce contexte, l'utilisation du point-en-haut (') ne se lie pas tant au fait qu'il s'agit ici d'un mot grec («andipera» $=\alpha v \tau i ́ \pi \varepsilon \rho \alpha$ ), qu'au fait que Solomos cherche à marquer la césure qui sépare les deux hémistiches. Et cela constitue une preuve supplémentaire de l'«intentionnalité métrique » inhérente à cet énoncé.

27. Massimo Peri, La «femme vêtue de lune» de Solomos, op. cit., p. 25. 28. Gérard Genette, Palimpsestes. La littérature au second degré, Paris, Éditions du Seuil, coll. «Poétique», 1982, p. 254-257.

29. Massimo Peri, La «femme vêtue de lune» de Solomos, op. cit., p. 25, note 16 . 
solomiennes. Nous espérons avoir montré, au contraire, que l'italien interagit avec le grec à différents niveaux tout au long de la genèse des Libres Assiégés. Ainsi, loin d'une vision réductrice qui conçoit l'italien comme la langue de la conception et le grec comme celle de l'exécution, il serait plus approprié de considérer leur incessante interaction comme un dialogue créatif entre les deux langues stimulant la production littéraire d'un poète bilingue. De manière plus générale, Solomos tente de créer un grec littéraire moderne qui, bien que proche du parler populaire grec de l'époque, soit capable d'exprimer avec précision des idées profondes, en l'insérant également dans la longue tradition littéraire italienne. Il s'agit donc d'une relation étroite - bien que parfois latente - d'interdépendance et de complémentarité entre l'italien et le grec, dont résulte tout un panorama de pratiques d'écriture que nous avons détaillées plus haut.

Auteur d'une thèse sur la genèse des Libres Assiégés de Dionysios Solomos (Université de Paris IV, janvier 2012), Kostis PAVLOU enseigne la littérature néo-hellénique à l'Open University of Cyprus. Il est chercheur associé à l'ITEM (équipe «Multilinguisme, traduction, création»). Ses travaux portent surtout sur la littérature néo-hellénique et comparée, sur l'approche génétique de l'œuvre de Dionysios Solomos (1798-1857), sur le plurilinguisme littéraire, ainsi que sur la métrique.

kopavlou@yahoo.com 